\title{
A VGI Quality Assessment Method for VGI based on Trustworthiness
} for VGlbased on Trustworthiness

\author{
Amir Masoud Forati and Farid Karimipour \\ University of Tehran, Tehran, Iran
}

\begin{abstract}
Recent advances in technology have greatly transformed how geographic information is produced and have led to the phenomenon of Volunteered Geographic Information (VGI). VGI allows people with little geographical knowledge to contribute in the creation of maps and other kinds of geographic information. Because $V G I$ is gathered by individuals who often have no formal training, the credibility and reliability of VGI is challenging. In this paper, we study what kinds of things might contribute to an assessment of the trustworthiness of data and the reputation of contributors in a VGI system. We present a model for analysing these characteristics and a method for automatically creating trustworthiness and reputation scores in order to assess the quality of VGI features.
\end{abstract}

\section{Keywords:}

Volunteered Geographic Information (VGI), data quality, trustworthiness, user's reputation

\section{Introduction}

Volunteered Geographic Information (VGI) is an approach to crowdsource information about geospatial features around us. Recently, VGI has gained increasing attention, and (web) services relying on it are becoming ubiquitous (D'Antonio et al., 2014).

Positional, thematic and temporal accuracy, logical consistency and completeness are the elements that ISO/TC 211 laid down and standardized in 2002 for the assessment of spatial data credibility (ISO, 2002). We face the issue of heterogeneous credibility because of the collaborative environment of VGI, in which content is created in an arbitrary process with little or no centralized control of the virtual communities involved, by users who are often non-experts, specifically not geospatial information experts. In addition, spatial entities are naturally ambiguous. Moreover, any user has his/her own perception, conceptualization, cultural background and spatial enablement. Thus, the existence of large number of VGI users with diverse motivations and backgrounds makes standardizing the credibility of geospatial information no mean feat. All in all, we need to use VGI-specific methods to assess the credibility of this kind of information.

A basic method to assess the credibility of a VGI dataset is to compare it against an authoritative dataset that was produced professionally. This method has two major 
drawbacks, however. First, an authoritative dataset is sometimes unavailable or may be very expensive. Second, this means of assessing quality is not universally valid. We therefore need to use alternative methods to assess the credibility of VGI. A provenance-based model to assess the quality of VGI, which would take these issues into account, was proposed by Bishr \& Janowicz (2010). In their method, quality assessment is based on an analysis of each feature's editing history in order to allocate a degree of trustworthiness to the feature for the 'data trustor' (i.e. the VGI user). Trust is a primary aspect of social networks, and it has been shown that it plays a major role in online communities (Golbeck, 2005).

Intuitive concepts such as the many-eyes principle (Haklay et al., 2010) are the basis of the trust analysis. This principle means that, if features are edited by a high number of users, the quality of these features is enhanced. Bishr \& Kuhn (2013) propose that trust holds only between people. For example, trusting a company like an insurance company to compensate our financial losses is, in fact, to trust the company's management and staff - in other words, the people behind the company. So it could be derived from this that trust in features is based on trust in the users' responsibility for these features.

Interpersonal trust implies the transition of trust from the person who is trusted to the information which that person conveys. The trustor can then assert trust directly in the information. This can be called informational-trust, where a trusting tie between a trustor and information such as VGI is mediated by interpersonal trust between the VGI originator and the VGI consumer (Keßler \& de Groot, 2013).

We use trust in social networks as a foundation for our alternative credibility assessment approach. In fact, trust in social networks is a measure of how information produced by some users is relatively valuable to others. Indeed, the more useful information is produced by more trusted users (Bégin et al., 2013; Haklay et al., 2010; Neis \& Zipf, 2012). We draw inspiration from this (Keßler \& de Groot, 2013) and extend it by relating data trustworthiness, user reputation, and social group reputation in order to assess VGI more properly.

\section{Related Work}

As suggested by Flanagin \& Metzger (2008), there is a critical need for the identification of methods and techniques to evaluate VGI quality. Quality assessment of VGI is still a fairly new research issue.

Bishr \& Janowicz (2010) used informational trust as a proxy measure for quality. Their proposal was one of the first to use trustworthiness for quality assessment of VGI. Keßler et al. (2011) apply Bishr \& Janowicz's use of trust as a proxy measure, by introducing a provenance vocabulary for OpenStreetMap (a provenance vocabulary allows the lineage of any online data to be made explicit, e.g. Created by, Creation Guideline or tag), and by showing how to annotate OpenStreetMap (OSM) data using this vocabulary. Their method lays the foundation for approaches to assess data quality that build solely on the information collected in the OSM database, using the trustworthiness of features as a proxy function for data quality. Keßler \& de Groot (2013) used trust and provenance for studying contribution 
patterns in the case of OSM, and suggested a number of indicators that have an impact on trust and that were basically derived from data provenance. They used five parameters for trustworthiness evaluation: (1) Versions, (2) Users, (3) Confirmations, (4) Tag corrections and (5) Rollbacks. These parameters make quality assessment possible without comparison to references. Trustworthiness is associated with each feature and represents the proxy value of data quality. Keßler \& de Groot (2013) introduced the question of user reputation, but leave it for future refinement. Using OSM as a case study, Ramos et al. (2013) worked on a method that handles semantic heterogeneity when VGI data is integrated into an authoritative dataset. Vandecasteele \& Devillers (2013) improved VGI quality by using measurements for semantic similarity; this enhances the user's experience by automatically suggesting attributes or by detecting attributes that are not related to each other. D'Antonio et al. (2014) introduced a model to evaluate VGI user reputation and VGI feature trustworthiness as proxy measures for data quality. Forati et al. (2015) include semantics in the crowdsourced data gained from a landscape description that used VGI. Their results show that there is a relation between VGI users' semantic characteristics, such as their occupation, and the quality of the features. Inspired by their work, we propose an algorithm which takes users' characteristics and their psychosocial parameters into account to assess the quality of any VGI system.

\section{$3 \quad$ Model overview}

Information that a user enters into a VGI System reflects his/her personality, and the collection of that information creates the user's reputation in the VGI system. In fact, we see reputation as the perception of trustworthiness of a person by a community (Goodchild, 2007). The reputation of a person belongs to the community and depends on many social and psychological factors, such as previous behaviour, community perception of the person, and the capacity of the community to sanction bad behaviour. Psychological factors include for example gender, age, culture and occupation. We propose a model which evaluates the VGI's level of trustworthiness by assessing the reputation of its user and of his/her social group. In fact, reputation helps the trustor to assess how reliable the trustee is likely to be (Bishr \& Janowicz, 2010). In other words, our model assesses the quality of VGI data through a proxy measure: trustworthiness.

Trustworthiness is defined as a 'bet about the future contingent action of others' (Sztompka, 1999, p. 25). In this sense, trustworthiness is strictly related to the concept of (others') reputation and social group reputation. We can estimate future contingent action of others by using these two parameters. Taking five parameters introduced by Kessler \& de Groot (2013), we can assign a degree of trust to each feature's version. All the features thus have a degree of trust. These parameters are the basis for the calculation of reputation. In fact, user reputation depends on the trustworthiness of all the feature versions edited by users and is defined as the average of such values:

$$
R(u . t)=\frac{\sum_{f_{i} \in F(u . t)} T\left(f_{i}\right)}{|F(u . t)|}
$$


where $F(u, t)$ is the set of all the feature versions edited by user $u$ until time $t$.

Accreditation of the users who have no history of performance, and rectification of current users' credibility are done by considering social group reputation, because most people's actions and reactions are influenced by social elements, such as their gender, their occupation, the environment in which they grew up, and their culture. If we group the users according to elements like gender, occupation and age, we can revise the users' reputations. In fact, when a feature is created or edited by a user, our anticipation of user reputation is based on his/her social group's reputation. This model is a self-improvement model: the more user statistics we have, the more reliable the quality assessment. So the model improves over time with increased numbers of users, and as a byproduct we can draw conclusions about the spatial cognition of different social groups.

The flowchart in Figure 1 shows how the VGI quality assessment method that we propose functions. The output of this method is the degree of trust for each of the features, which consists of the version quality of a feature, the user's performance history and related reputation, and the reputation of the editor's social group. Initially, the user's performance history will be checked. If there is no history of performance for the feature editor (the editor is a newcomer), we take the editor's social group reputation into account in order to obtain a better and more reliable degree of trust. Assume that the user is a member of a social group almost all of whose edits have a high degree of trust: our anticipation is that the degree of trust of a newcomer's edit reflects the overall degree of trust of other members of that social group. This means that the quality of his/her edits is similar to that of the edits made by the social group as a whole.

If the user does not fulfil our anticipation and his/her edits fall below the social group's overall trust, with time the other trust elements of his/her edited feature decline, and consequently that feature's degree of trust decreases. However, because newcomers' performances modify the social group's overall quality, this leads us to more reliable quality assessment. 


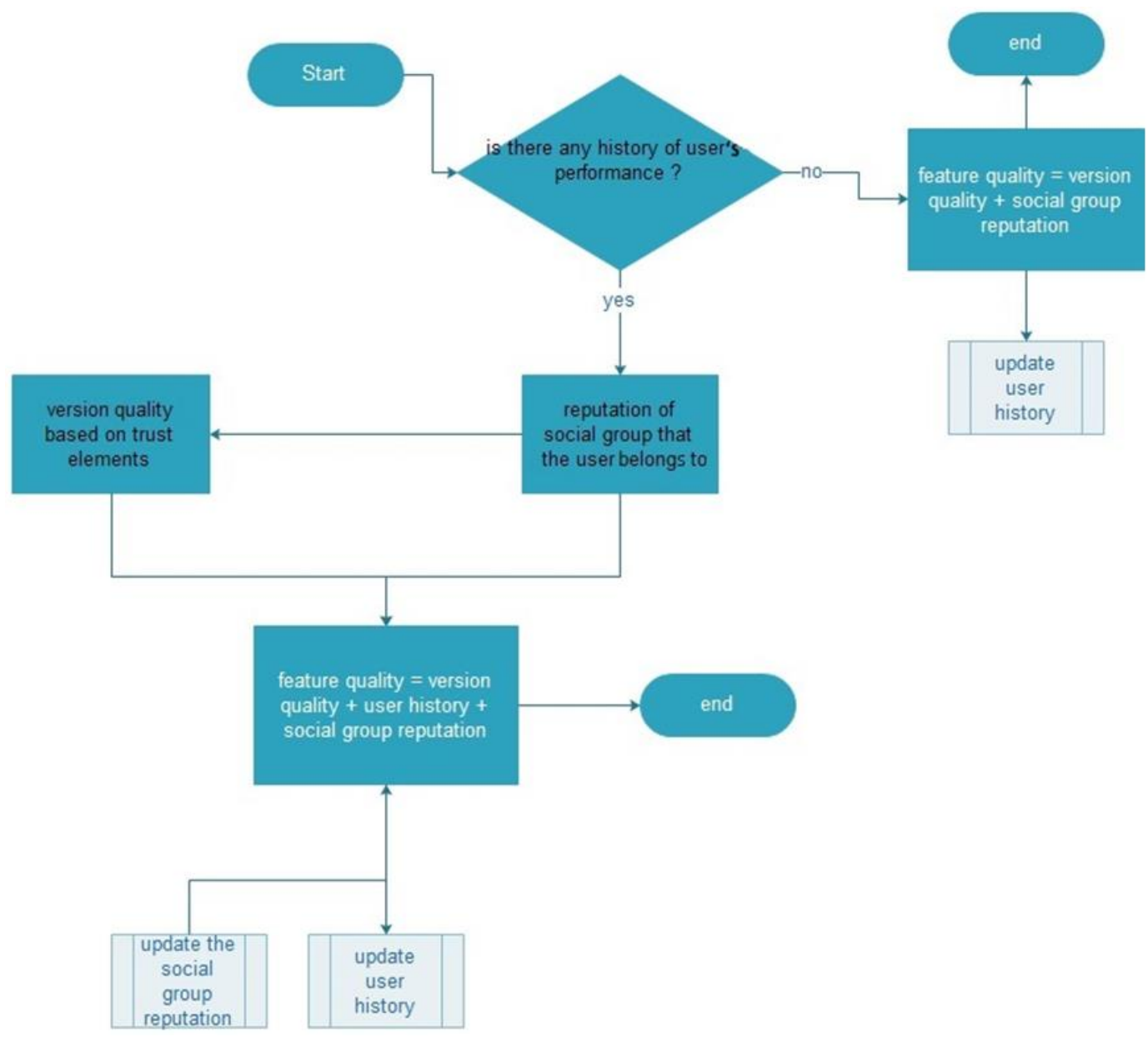

Figure 1: Flowchart for the proposed algorithm

\section{$4 \quad$ Implementation}

In order to examine our proposed algorithm, we developed a VGI system using the Android platform that aims at collecting landscape description information. Landscape refers to the visible features of an area of land, often considered in terms of their aesthetic appeal. Landscape description provides insight into the worlds of perception. Knowing how people see and perceive a landscape imparts additional information about different characteristics of a landscape that could be used in aesthetic analysis, environmental protection, an examination of visual pollution, wayfinding and landscaping (Forati et al., 2015).

In our system, three psychosocial criteria are used for user-grouping: age, gender and occupation; the quality assessment for VGI is carried out under these groups. In other words, we expect that users of the same age, gender and occupation will perform in roughly similar fashion, and so the quality of the data that these people provide will be similar. 
Gender affects the attitude and personal ability of users (Goldhaber et al., 2014). Their age can give us an estimate of how much experience they have. It is clear that user experience plays a significant role in providing high-quality VGI. And finally, users' occupations give an indication of their levels of computer literacy and spatial abilities, so these features, too, have a direct impact on the quality of the information the users produce.

To describe a landscape, a few metrics are required. Given that our aim was to discover users' spatial ability and their peripheral perception, we chose four metrics:

1. The main feature of the landscape

2. Distance from the main feature: estimated distance between the user's location (camera position) and the main feature; the actual distance is calculated, as is the deviation of the measured distance from estimated distance.

These two metrics determine the spatial ability of the user.

3. The landscape's dominant colour: this is determined using a histogram for landscape colour (a colour histogram shows the proportion of pixels of each colour within the image), which is compared with the colour identified by the user.

4. The landscape's quality of being eye-catching: scored from 0 to 100 .

The last two metrics determine the users' peripheral perceptions.

After the implementation of the system, 1,000 users participated in the project. The results and data obtained were reviewed and analysed statistically. In order to calculate the level of consensus in a social group with regard to numerical variables, we used the standard deviation. The results of the statistical analysis and the standard deviations obtained clearly point to the effectiveness of the proposed algorithm. Various results of the analysis are shown in Figures 2, 3, 4 and 5.

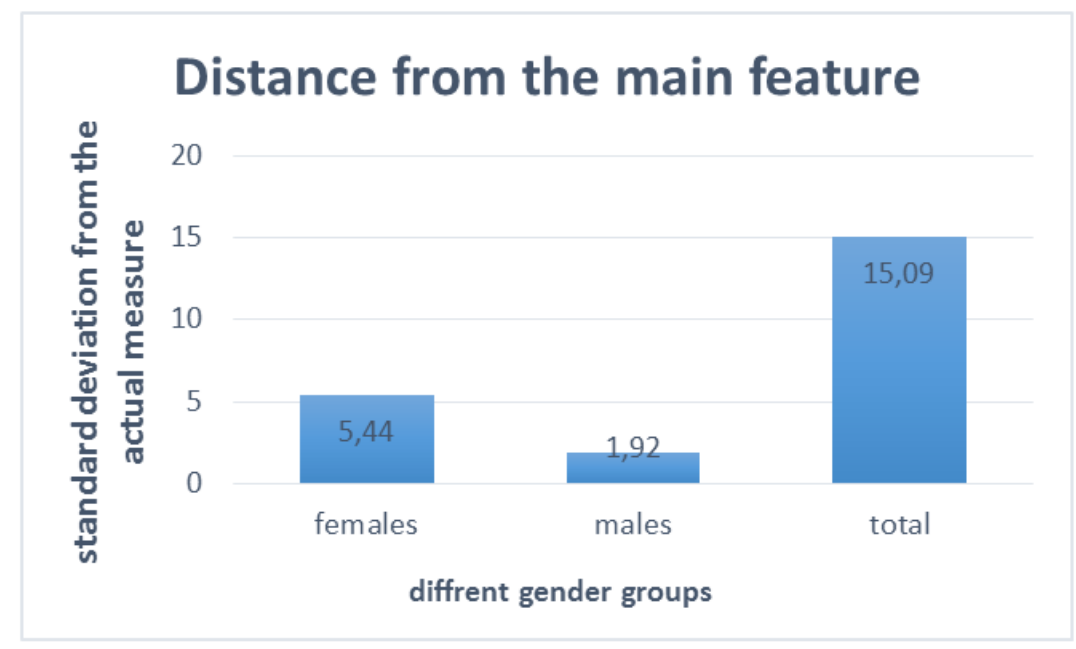

Figure 2: The correlation between the perceived distance from the main feature and the user's gender 


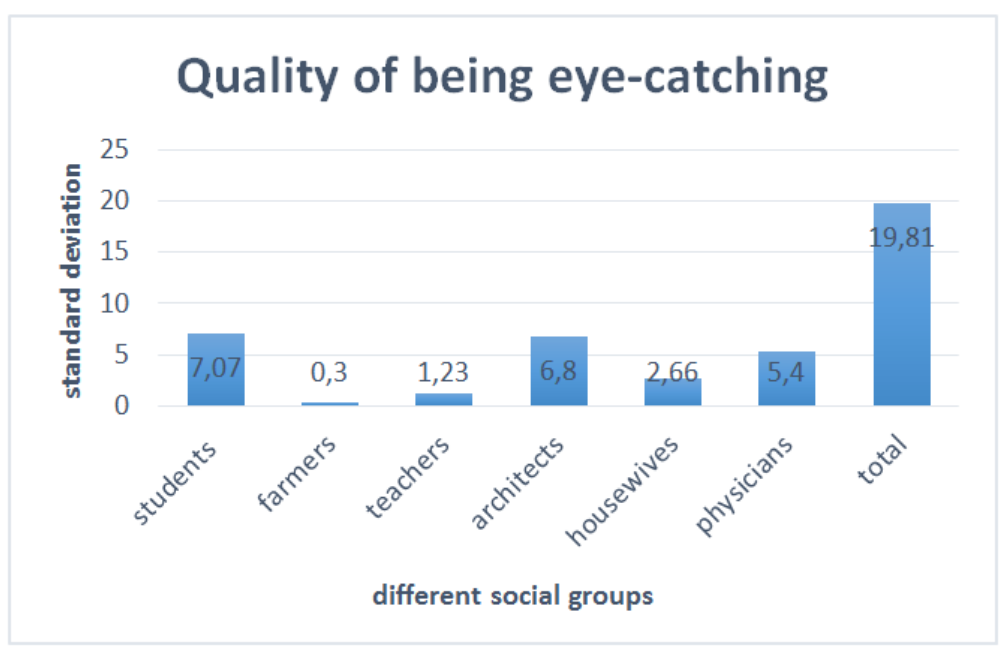

Figure 3: The correlation between how eye-catching a landscape is and the user's occupation

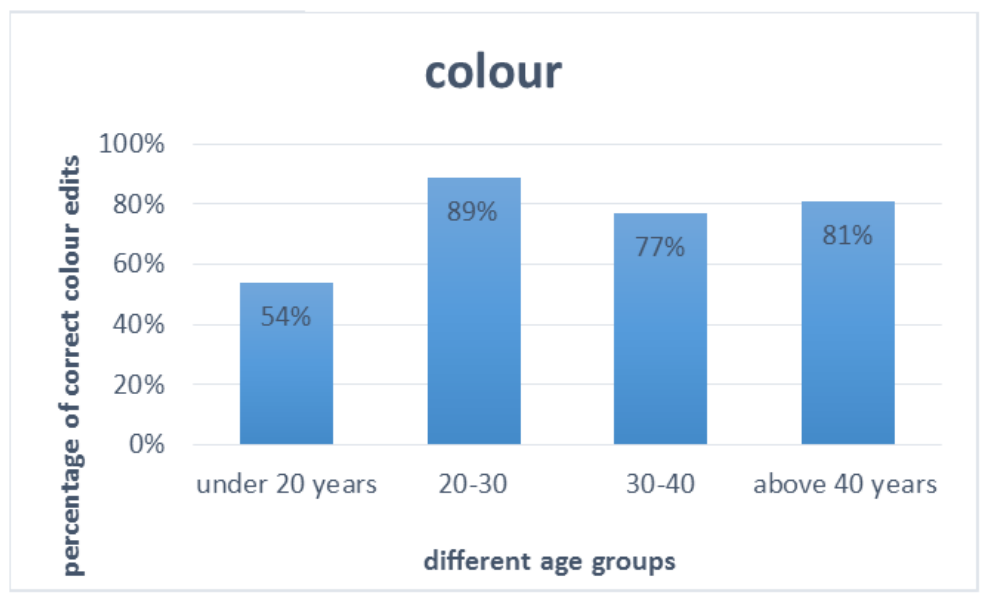

Figure 4: The correlation between the perceived dominant colour and the user's age 


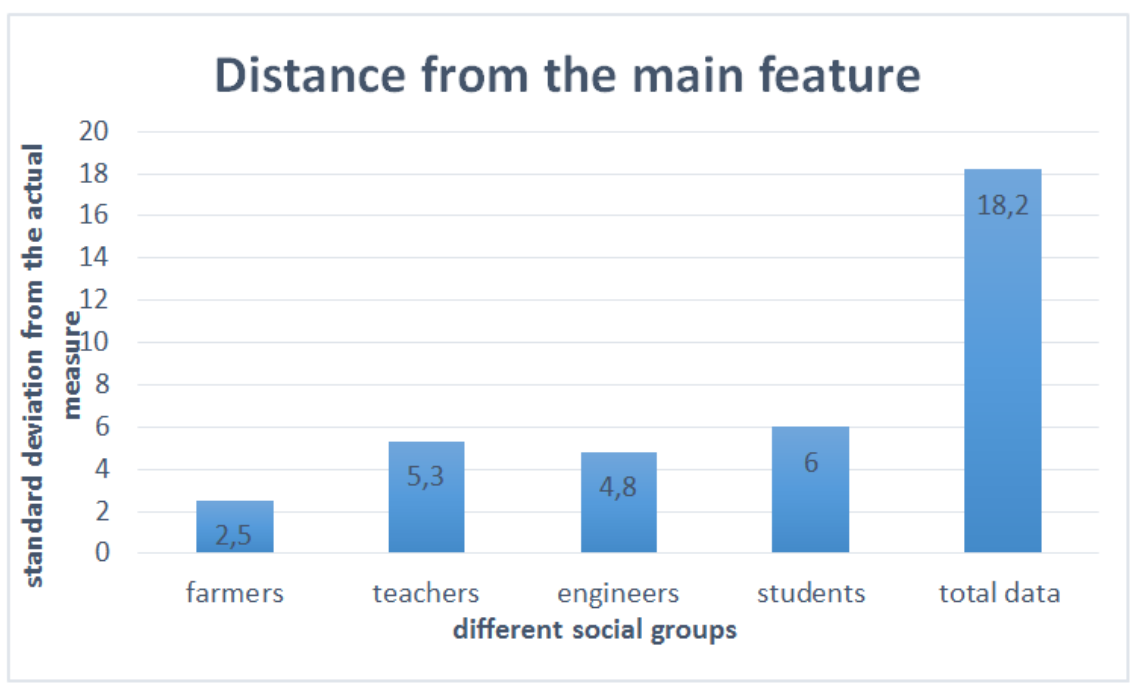

Figure 5: The correlation between the perceived distance from the main feature and the user's occupation

As can be seen in Figures 2-5, there is a significant difference between the standard deviation for all data combined and for individual social groups. This highlights that the users acted according to our expectations. Finally, it is worth mentioning that there is a direct relationship between the algorithm results and the number of participants; in other words, the higher the number of participants, the more reliable the quality assessment.

\section{Conclusion}

This paper proposes an approach to include the user's reputation and their psychosocial factors in evaluating the credibility of VGI. The initial outcome of implementing the approach shows an improvement in the final results, and the process will improve by itself over time. The main concerns for future work are grouping and assigning initial credibility values. We are also working on new elements that impact the spatial ability of users, which could also be taken into consideration in VGI quality assessment.

\section{References}

Bégin, D., Devillers, R., \& Roche, S. (2013). Assessing volunteered geographic information (VGI) quality based on contributors' mapping behaviours. Paper presented at the Proceedings of the 8th international symposium on spatial data quality ISSDQ.

Bishr, M., \& Janowicz, K. (2010). Can we trust information?-the case of volunteered geographic information. Paper presented at the Towards Digital Earth Search Discover and Share Geospatial Data Workshop at Future Internet Symposium, volume. 
Bishr, M., \& Kuhn, W. (2013). Trust and reputation models for quality assessment of human sensor observations Spatial Information Theory (pp. 53-73): Springer.

D’Antonio, F., Fogliaroni, P., \& Kauppinen, T. (2014). VGI edit history reveals data trustworthiness and user reputation. Paper presented at the 17th AGILE International Conference on Geographic Information Science (Short Paper).

Flanagin, A. J., \& Metzger, M. J. (2008). The credibility of volunteered geographic information. GeoJournal, 72(3-4), 137-148.

Forati, A., Soleimani, S., Karimipour, F., \& Malek, M. (2015). INCLUDING USERS'SEMANTICS IN EVALUATING THE CREDIBILITY OF CROWDSOURCED LANDSCAPE DESCRIPTIONS. The International Archives of Photogrammetry, Remote Sensing and Spatial Information Sciences, 40(3), 35.

Golbeck, J. A. (2005). Computing and applying trust in web-based social networks.

Goldhaber, T., Langdon, P., \& Clarkson, P. (2014). Gender Issues in ICT Adoption: A Literature Review Inclusive Designing (pp. 59-67): Springer.

Goodchild, M. F. (2007). Citizens as sensors: the world of volunteered geography. GeoJournal, 69(4), 211-221.

Haklay, M., Basiouka, S., Antoniou, V., \& Ather, A. (2010). How many volunteers does it take to map an area well? The validity of Linus' law to volunteered geographic information. The Cartographic Journal, 47(4), 315-322.

ISO, I. (2002). 19113-Geographic Information-Quality Principles. International Organization for Standardization.

Keßler, C., \& de Groot, R. T. A. (2013). Trust as a proxy measure for the quality of volunteered geographic information in the case of OpenStreetMap Geographic information science at the heart of Europe (pp. 21-37): Springer.

Keßler, C., Trame, J., \& Kauppinen, T. (2011). Tracking editing processes in volunteered geographic information: The case of OpenStreetMap. Paper presented at the Identifying objects, processes and events in spatio-temporally distributed data (IOPE), workshop at conference on spatial information theory.

Neis, P., \& Zipf, A. (2012). Analyzing the contributor activity of a volunteered geographic information project-The case of OpenStreetMap. ISPRS International Journal of Geo-Information, 1(2), 146165.

Ramos, J. M., Vandecasteele, A., \& Devillers, R. (2013). Semantic integration of authoritative and volunteered geographic information (VGI) using ontologies. Paper presented at the Proceeding of Association of Geographic Information Laboratories for Europe (AGILE) Conference 2013.

Sztompka, P. (1999). Trust: A sociological theory: Cambridge University Press.

Vandecasteele, A., \& Devillers, R. (2013). Improving volunteered geographic data quality using semantic similarity measurements. ISPRS-International Archives of the Photogrammetry, Remote Sensing and Spatial Information Sciences, 1(1), 143-148. 